\title{
Molecular dynamics of ribosomal elongation factors G and $\mathrm{Tu}$
}

\author{
Katarzyna Kulczycka • Maciej Długosz • \\ Joanna Trylska
}

Received: 27 August 2010/Revised: 9 November 2010/Accepted: 16 November 2010/Published online: 9 December 2010

(C) The Author(s) 2010. This article is published with open access at Springerlink.com

\begin{abstract}
Translation on the ribosome is controlled by external factors. During polypeptide lengthening, elongation factors EF-Tu and EF-G consecutively interact with the bacterial ribosome. EF-Tu binds and delivers an aminoacyl-tRNA to the ribosomal A site and EF-G helps translocate the tRNAs between their binding sites after the peptide bond is formed. These processes occur at the expense of GTP. EF-Tu:tRNA and EF-G are of similar shape, share a common binding site, and undergo large conformational changes on interaction with the ribosome. To characterize the internal motion of these two elongation factors, we used $25 \mathrm{~ns}$ long all-atom molecular dynamics simulations. We observed enhanced mobility of EF-G domains III, IV, and V and of tRNA in the EF-Tu:tRNA complex. EF-Tu:GDP complex acquired a configuration different from that found in the crystal structure of EF-Tu with a GTP analogue, showing conformational changes in the switch I and II regions. The calculated electrostatic properties of elongation factors showed no global similarity even though matching electrostatic surface patches were found around the domain I that contacts the ribosome, and in the GDP/GTP binding region.
\end{abstract}

Keywords Bacterial translation - Ribosome - Elongation factors $\mathrm{G}$ and $\mathrm{Tu} \cdot$ Molecular dynamics $\cdot$ Electrostatics

K. Kulczycka $\cdot$ M. Długosz $\cdot$ J. Trylska $(\bowtie)$

Interdisciplinary Centre for Mathematical and Computational

Modelling, University of Warsaw, Pawinskiego 5A,

02-106 Warsaw, Poland

e-mail: joanna@icm.edu.pl

K. Kulczycka

College of Inter-Faculty Individual Studies in Mathematics and Natural Science, University of Warsaw, Zwirki i Wigury 93, 02-089 Warsaw, Poland

\section{Introduction}

Translation is the process of converting the information stored in the nucleotide sequence of messenger RNA (mRNA) into the sequence of amino acids in a polypeptide. In prokaryotes and eukaryotes, translation takes place on ribosomes that are macromolecular complexes composed of ribosomal RNA and proteins. The ribosomes can be separated into two subunits, a large (in bacteria called 50S) and a small (30S) subunit (Liljas 2004). The 30S subunit binds mRNA and is responsible for the fidelity of translation. There are three inter-subunit binding sites for transfer RNAs (tRNAs) which are named A, P, and E. The A site is the point of entry for the aminoacyl-tRNA (aa-tRNA). The $\mathrm{P}$ site is where the peptidyl-tRNA is formed, and the E site is the exit site of the uncharged tRNA after it donates its amino acid to the growing peptide chain.

A number of protein factors that bind to the ribosome are involved in translation (Liljas 2004). Some of these are required for proper initiation or termination of protein synthesis. Others assist in the peptide elongation phase incorporating the aa-tRNAs or facilitating the ratchet-like motion of the ribosome required for translocation of tRNAs from the A site to the P site (Frank and Agrawal 2000). Many factors bind transiently to the ribosome and hydrolyse GTP. Therefore, they belong to the family of GTPases or G-proteins.

In prokaryotes, three elongation factors are involved in the peptide elongation process: $\mathrm{Tu}$ (EF-Tu), Ts (EF-Ts), and $\mathrm{G}(\mathrm{EF}-\mathrm{G})$. EF-Tu and EF-G are the members of the $\mathrm{G}$ protein family that has a conserved and common structural design. These two factors bind consecutively to the ribosomal sarcin-ricin loop and GTPase-associated centre located between the 50S and 30S subunits. EF-Tu is responsible for positioning the incoming aa-tRNA in the ribosomal A-site. 
First, the aa-tRNA binds to the active form of EF-Tu (EF-Tu complexed with GTP) (Kjeldgaard et al. 1993) and forms the ternary complex EF-Tu:GTP:aa-tRNA (Nissen et al. 1995). When the aa-tRNA anti-codon and mRNA codon recognition criteria are satisfied, a series of events leads to the accommodation of the aa-tRNA in the ribosomal A site. The hydrolysis of GTP to GDP drives a conformational change in EF-Tu, aa-tRNA is released from EF-Tu, and the EF-Tu:GDP complex dissociates from the ribosome (Rodnina et al. 1995; Polekhina et al. 1996). The EF-Ts elongation factor serves as the guanine nucleotide exchange factor for EF-Tu, catalysing the release of GDP. This enables EF-Tu to bind a new GTP molecule, release EF-Ts, and catalyse another addition of aa-tRNA to the ribosomal A-site (Kawashima et al. 1996; Jeppesen et al. 2005).

After the cognate aa-tRNA is placed in the A site, a peptide bond is formed between amino acids of the A site tRNA and P site tRNA, and EF-G complexed with GTP binds to the ribosome (Hansson et al. 2005). EF-G association with the ribosome, and GTP hydrolysis, help translocate the already unloaded tRNA from the A site to the $\mathrm{P}$ site, the peptidyl-tRNA from the $\mathrm{P}$ site to the $\mathrm{E}$ site, and the mRNA through the 30S subunit by a distance of one codon. Ticu et al. (2009) have shown that the GTP hydrolysis and the process of translocation are partially coupled. During translocation of tRNAs the ribosome undergoes a ratcheting motion (Agrawal et al. 1999; Stark et al. 2000; Frank and Agrawal 2000). After the translocation, the complex between EF-G and GDP (Czworkowski et al. 1994; Laurberg et al. 2000) dissociates and the ribosome is ready to accept the next aa-tRNA into the A site. The subsequent substitution of GDP for GTP by a Gnucleotide exchange factor reactivates EF-G.

EF-G is composed of five domains and EF-Tu of three (Fig. 1). Domain I (or G) of both factors contains the GTP (or GDP) binding site and domain II forms a unique $\beta$ barrel. The I domain of EF-G includes an insertion-the $\mathrm{G}^{\prime}$ subdomain - that is exclusive for EF-G and does not exist in EF-Tu. The three-dimensional structure of EF-G closely resembles that of the complex between EF-Tu and tRNA (Fig. 1). Both structures are of elongated shape. EF-G domains I and II are homologous with EF-Tu, and EF-G domains III, IV, and V adopt the shape of tRNA (Gudkov 2001). Such structural correspondence of molecular fragments is an example of molecular mimicry (Nyborg et al. 1996); the protein domains evolved so that they can mimic the shape of the tRNA molecule. The crystal structure of the GDP-inactive form of EF-G resembles the GTP-active structure of the ternary complex (Nyborg et al. 1996; Sergiev et al. 2005). EF-G and the ternary complex bind at the interface of the ribosomal subunits in an elongated but tight cleft close to the L11 protein of the 50S subunit (Gao et al. 2009; Schmeing et al. 2009).
The flexibility of elongation factors and free tRNA, and of tRNA bound to the ribosome, have been investigated using both experimental (Johanson et al. 1996; Martemyanov and Gudkov 1999, 2000; Vogeley et al. 2001; Schmeing et al. 2009; Gao et al. 2009) and theoretical (Tama et al. 2004; Wriggers et al. 2000; Sanbonmatsu et al. 2005; Li and Frank 2007; Spasic et al. 2008; Eargle et al. 2008) methods. Sanbonmatsu et al. (2005) applied all-atom molecular dynamics (MD) simulations of the entire ribosome in explicit solvent to investigate the last stages of incorporating tRNA into the ribosomal A-site. Li and Frank (2007) described the flexibility and the conformation of tRNA on the ribosome with all-atom MD and compared it with a cryo-electron microscopy map. They found the kink and twist conformations of the tRNA anticodon stem and described the detailed interactions between the tRNA and the ribosome. In the work of Spasic et al. (2008) a coarse-grained model was used to investigate the flexibility of the ternary complex relative to tRNA. It was shown that an isolated tRNA is more stable than the tRNA in the complex with EF-Tu. The authors also described the motions of the ribosome on association with the ternary complex based on fluorescence resonance energy transfer experiments and presented the nature and the sites of
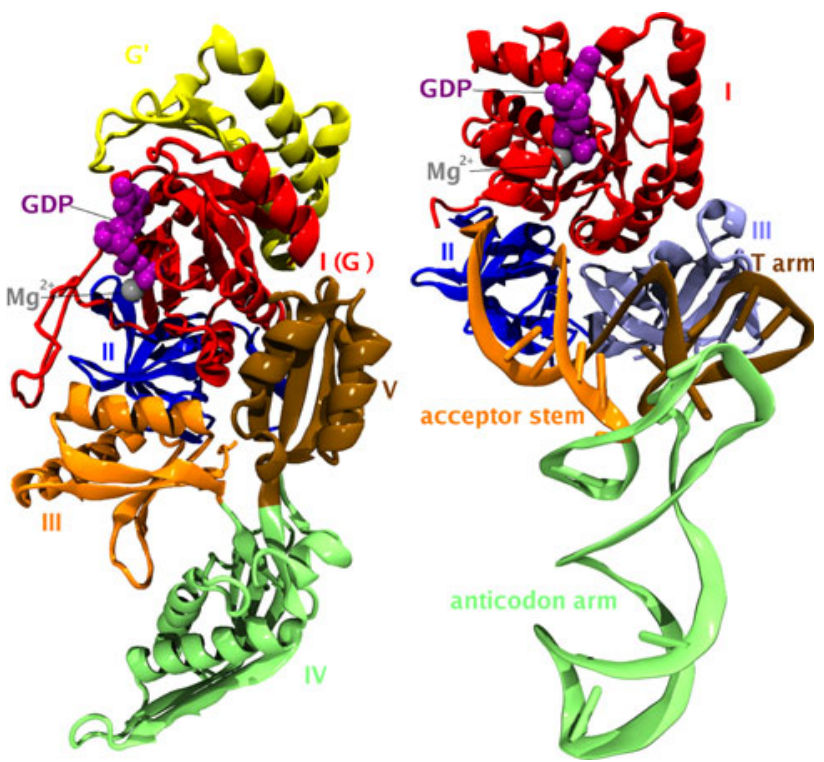

Fig. 1 Left: Secondary structure model of EF-G in the complex with GDP and $\mathrm{Mg}^{2+}$ (PDB code $1 \mathrm{FNM}$ ); domain $\mathrm{I}(\mathrm{G})$, residues $1-280$ (red); insert $\left(\mathrm{G}^{\prime}\right)$, residues 158-253 (yellow); domain II, residues 289-391 (dark blue); domain III, residues 404-482 (orange); domain IV, residues 483-603, 675-689 (green); domain V, residues 604-673 (brown). Right: EF-Tu complexed with aa-tRNA, GDP, and $\mathrm{Mg}^{2+}$ (based on the PDB code 1TTT); domain $\mathrm{I}(\mathrm{G})$, residues 1-211 (red); domain II, residues 220-311 (dark blue); domain III, residues 312-405 (light blue); aa-tRNA is divided into the acceptor stem (orange), anticodon arm (green), and T-arm (brown). Domains $\mathrm{I}(\mathrm{G})$ and II of EF-Tu and EF-G are homologous and common for GTPases 
binding of $\mathrm{Mg}^{2+}$ ions to the tRNA. In the work of Eargle et al. (2008) MD was applied to examine the flexibility of the EF-Tu-GTP-aa-tRNA ${ }^{\text {Cys }}$ complex and the role of $\mathrm{Mg}^{2+}$ ions and tRNA-modified bases. The authors proved that the ionic concentration affects not only the structure but also the dynamics and the binding of EF-Tu to tRNA. In other studies (Tama et al. 2004; Wriggers et al. 2000), the authors used cryo-electron microscopy maps to combine structural information with functionally important dynamic properties of EF-G. In both cases they reproduced the conformational changes of EF-G upon its complexation with the ribosome.

In this work we focussed on comparing the internal movements and electrostatic properties of elongation factors that associate with the ribosome. We performed $25 \mathrm{~ns}$ long all-atom MD simulations in implicit solvent to investigate the internal dynamics of EF-G and EF-Tu with aa-tRNA. The simulations for the following systems were performed: EF-G, EF-G in the complex with GDP and $\mathrm{Mg}^{2+}, \mathrm{EF}-\mathrm{Tu}, \mathrm{EF}-\mathrm{Tu}$ complexed with GDP and $\mathrm{Mg}^{2+}$, and EF-Tu in the complex with aa-tRNA. We examined whether there is any relationship between the structural similarity of EF-G and EF-Tu complexed with aa-tRNA and their overall dynamics. We have shown that these systems are characterized not only by the similar shape and binding position on the ribosome but also by similar dynamical behavior with the most significant motions observed for their corresponding elongated parts. We also simulated EFTu without its aa-tRNA to elucidate the conformational freedom of the EF-Tu in its GDP form when it needs to dissociate the tRNA molecule and leave the ribosome. We also compared the electrostatic properties of the studied elongation factors and found no global electrostatic similarity.

\section{Methods}

Preparation of structures

The crystal structures of EF-G or EF-Tu complexed with GTP are not available in the Protein Data Bank. For the EF-G study we chose the crystal structure of EF-G from Thermus thermophilus with guanosine-5'-diphosphate and the $\mathrm{Mg}^{2+}$ ion located near the $\beta$-phosphate group of GDP (PDB code 1FNM (Laurberg et al. 2000), resolution $2.8 \AA$ ). The structures of native EF-G complexed with GDP and a mutant EF-G complexed with a GTP analogue were found to be similar (Hansson et al. 2005). Because the 1FNM structure was incomplete, the missing amino acids (residues: 4, 5, 40-67 and 689-691) were added. Their positions were energy optimized in vacuum with the steepest descent and L-BFGS methods using the GROMACS package (Berendsen et al. 1995). Two variants of the EF-G system were prepared (Fig. 1): EF-G complexed with one GDP molecule and $\mathrm{Mg}^{2+}$ and its apo form (EF-G without GDP and $\mathrm{Mg}^{2+}$ ).

In case of EF-Tu, the crystal structure of the complex between EF-Tu, tRNA ${ }^{\text {Phe }}$, guanosine-5'-( $\beta, \gamma$-imido $)$ triphosphate (GDPNP) and the $\mathrm{Mg}^{2+}$ ion from Thermus aquaticus (PDB code 1TTT (Nissen et al. 1995), resolution $2.7 \AA$ ) was used. GDPNP is a nonhydrolysable GTP analogue which was used in crystallography to prevent the hydrolysis of GTP to GDP. During this hydrolysis, EF-Tu was found to undergo a conformational change (Abel et al. 1996; Polekhina et al. 1996; Nissen et al. 2000). We replaced the GTP analogue in the initial EF-Tu structure with GDP; in this way we wanted to trigger a conformational transition in EF-Tu. Three variants of the EF-Tu system were constructed (Fig. 1): EF-Tu complexed with tRNA $^{\text {Phe }}$, EF-Tu with GDP and $\mathrm{Mg}^{2+}$, and free EF-Tu.

Overall, we present the results of five $25 \mathrm{~ns}$ long MD simulations that are labeled: EF-G, EF-G:GDP, EFTu:tRNA, EF-Tu, and EF-Tu:GDP. GDP is accompanied by the $\mathrm{Mg}^{2+}$ ion.

\section{MD simulations}

MD simulations were performed with the AMBER 9 package (Pearlman et al. 1995; Case et al. 2005, 2006) and the molecules were described according to the AMBER 2003 force-field (Duan et al. 2003; Lee and Duan 2004). The parameters for GDP were generated using the Antechamber module of AMBER 9. A modified generalized Born model (GB ${ }^{O B C}$ (Onufriev et al. 2004)) was used to describe the solvation effects. An implicit solvent model was selected because it enables faster sampling with less computational effort. Moreover, the interactions are screened by a continuum distribution of ions which can be an advantage if the positions of counter-ions are not resolved crystallographically (especially for EF-Tu:aatRNA which is the system with the largest negative net charge). It has previously been shown that the explicit and implicit solvent simulations are in reasonable agreement both for proteins and nucleic acids (Tsui and Case 2000; Shen and Freed 2002), see the discussion in the "Conclusions" section).

The solute and solvent dielectric constants were set to 1 and 80 , respectively. The cutoff distance for the non-bonded interactions was set to $18 \AA$. All simulations were conducted at $150 \mathrm{mM}$ ionic strength and at $293 \mathrm{~K}$. The Andersen scheme was used for temperature control (Andrea et al. 1983). The SHAKE algorithm (Ryckaert et al. 1977) was applied to all bonds involving hydrogen 
atoms allowing for a $1.5 \mathrm{fs}$ integration time step. Before MD simulations, all structures were energy-minimized with the steepest descent and conjugate gradient algorithms.

\section{Calculations of the electrostatic potential}

The electrostatic potential around EF-G and EF-Tu:tRNA complex was calculated using the implicit solvent PoissonBoltzmann model (Honig and Nicholls 1995). The AMBER force-field (Duan et al. 2003; Lee and Duan 2004) partial charges and radii were used for solutes. The solute and solvent dielectric constants were set to 4 and 78.5 , respectively. The boundary between the solute and the solvent was defined as the van der Waals surface. Monovalent ionic strength was set to $150 \mathrm{mM}$ and the Stern ion exclusion layer to $2.0 \AA$. The non-linear PoissonBoltzmann equation was solved numerically on a three dimensional grid with the Adaptive Poisson Boltzmann Solver (APBS; Baker et al. 2001). Solutions to a grid spacing of $0.35 \AA$ were reached.

\section{MD data analysis}

As a measure of the global dynamic properties, the root mean square deviation (RMSD), the root mean square fluctuation (RMSF), and the radius of gyration (GR) were analysed. The RMSD between structures was calculated as:

$\operatorname{RMSD}=\sqrt{\frac{1}{N} \sum_{i=1}^{N}\left(\mathbf{R}_{i}(t)-\mathbf{R}_{i}(0)\right)^{2}}$

where $N$ is the number of atoms, vectors $\mathbf{R}_{i}(t)$ and $\mathbf{R}_{i}(0)$ describe the Cartesian coordinates of atom $i$ in the instantaneous configurations at time $t$ and in the reference structure, respectively.

The RMSF was computed using the equation:

$\mathrm{RMSF}=\sqrt{\frac{1}{M} \sum_{i=1}^{M}\left(\mathbf{R}_{i}(t)-\left\langle\mathbf{R}_{i}\right\rangle\right)^{2}}$

where $M$ is the number of snapshots in the MD trajectory, and $\left\langle\mathbf{R}_{i}\right\rangle$ describes the Cartesian coordinates of an average structure.

The $\operatorname{GR}(t)$ was computed from the definition:

$\operatorname{GR}(t)=\frac{1}{2 N^{2}} \sqrt{\sum_{j=1}^{N} \sum_{i=1}^{N}\left(\mathbf{R}_{i}(t)-\mathbf{R}_{j}(t)\right)^{2}}$.

As a measure of inter-domain motions, we also calculated the distances between the centres of masses (COM) of elongation factor domains,
$d_{A, B}^{\mathrm{COM}}(t)=\sqrt{\left(\frac{\sum_{i=1}^{N_{A}} \mathbf{R}_{i}(t) m_{i}}{\sum_{i=1}^{N_{A}} m_{i}}-\frac{\sum_{j=1}^{N_{B}} \mathbf{R}_{j}(t) m_{j}}{\sum_{j=1}^{N_{B}} m_{j}}\right)^{2}}$

where $m$ describes the masses of atoms in a domain $(A$, $B)$ and $N_{A}, N_{B}$ are the numbers of atoms in the domains.

Principal-components analysis (PCA; Amadei et al. 1993), based on covariance matrix diagonalization, was carried out to identify the most significant modes of fluctuations in the systems and to describe their directions and range.

$M_{\text {covar }}=\left\langle\left[\mathbf{R}_{i}(t)-\left\langle\mathbf{R}_{i}(t)\right\rangle\right]\left[\mathbf{R}_{j}(t)-\left\langle\mathbf{R}_{j}(t)\right\rangle\right]\right\rangle$

where \langle\rangle indicates an MD average and $\mathbf{R}_{i}(t), \mathbf{R}_{j}(t)$ are the Cartesian coordinates of atoms $i, j$. Only $\mathrm{C}_{\alpha}$ and $\mathrm{P}$ atoms were used to construct the covariance matrices.

To cluster the MD conformations, we applied the Jarvis-Patrick method (Jarvis and Patrick 1973) implemented in the GROMACS package (Berendsen et al. 1995). The Jarvis-Patrick method of clustering is a technique based on the shared nearest neighbours algorithm. Two structures are assigned to the same cluster if they share a set threshold number of nearest neighbours. The two structures must also contain each other in that nearest neighbour list. Similarity between different structures is determined by using a distance metric, i.e. RMSD. Two parameters specific to the Jarvis-Patrick algorithm: the number of neighbours to be examined and the minimum required number of neighbours in common, were set to 10 and 3, respectively.

In all analyses, unless explicitly stated, the initial $1 \mathrm{~ns}$ trajectory data were discarded and the $24 \mathrm{~ns}$ production phase was used. Trajectories were analysed using the Ptraj module of AMBER9, the PCA was performed with GROMACS (Berendsen et al. 1995), visualization was carried out under VMD (Humphrey et al. 1996) and Chimera (Pettersen et al. 2004).

\section{Results and discussion}

Internal flexibility of EF-G and EF-Tu:tRNA

In MD simulations both EF-G and EF-Tu:tRNA have significant internal mobility. The radius of gyration, which is a measure of the size and the compactness of an object, changes in the explored time scale (Fig. 2, top). The initial radii of gyration corresponding to crystallographic structures are 30.8 and $29.4 \AA$ for EF-G and EF-Tu:tRNA, respectively. For EF-G the radius of gyration decreases during MD simulations and its fluctuations are related to the movements of the domain IV. For EF-Tu:tRNA the radius of gyration increases in comparison with the one 
calculated for the crystal structure and its changes result from the movement of tRNA.

The RMSD computed relative to the crystal structures is shown in Fig. 2 (bottom). For both systems, the RMSD becomes level after $1 \mathrm{~ns}$. However, considerable fluctuations (larger for EF-Tu:tRNA) are noticeable. We find that these fluctuations are related to large-scale movements of tRNA in the EF-Tu:tRNA complex and of the domain IV (i.e. the tail) of EF-G (Fig. 1). The interconversion between such "extended" and "folded" forms is characteristic for these systems and is related to their function (Liljas 2004) because upon binding to the ribosome the factors undergo extensive configurational changes (Agrawal et al. 1998; Valle et al. 2003; Tama et al. 2004; Wriggers et al. 2000; Ticu et al. 2009; Munro et al. 2010).

To discern the conformational movements in EF-G and EF-Tu:tRNA, we quantified the changes in RMSD between each trajectory frame. The RMSD-matrices and the cluster analysis are presented in Fig. 3. We observed several clusters with a time-span between 1.5 and $6 \mathrm{~ns} ; 16$ groups of different configurations for EF-G (Fig. 3), 22 groups for EF-G:GDP (data not shown), and 11 groups for EFTu:tRNA (Fig. 3). The average structures of the most numerous populations of EF-G and EF-Tu:tRNA are shown in Fig. 4. The average RMSD between the structures within each cluster is about $1 \AA$. The RMSD between structures from different clusters ranges from 2 to $3 \AA$. In both cases the most important structural differences between the generated clusters are in the elongated parts, i.e. domains III, IV, and V of EF-G and tRNA of the EF-
Tu:tRNA complex. The changes are in agreement with studies showing that the mobility of these fragments is important for the binding of the factors to the ribosome (Agrawal et al. 1998; Valle et al. 2003; Tama et al. 2004; Wriggers et al. 2000). Specifically, when tRNA is delivered by EF-Tu to the ribosome, the tRNA anticodon arm is distorted (Valle et al. 2002; Schuette et al. 2009).

To identify any transitions between the observed configurational states, we examined the distances between the centres of masses (COM) of various domains. COM distances as a function of the simulation time calculated for $\mathrm{C}_{\alpha}$ and $\mathrm{P}$ atoms are presented in Fig. 5. The largest changes in the COM distances (up to $10 \AA$ ) were observed between EF-G domains II and IV in both EF-G (Fig. 5a, black line) and EF-G:GDP (Fig. 5a, blue line). In the corresponding fragments, i.e. the domain II and tRNA of EF-Tu:tRNA, we observed similar (although smaller in magnitude) changes (Fig. 5a, red line). Both molecules undergo a transition from compact to "extended" forms involving domain IV of EF-G and tRNA (also described in the section on PCA analysis).

To investigate collective motion of EF-G and EFTu:tRNA, we performed PCA. As described in the "Methods" section, the covariance matrices corresponding to the $\mathrm{C}_{\alpha}$ and $\mathrm{P}$ coordinates were calculated and the eigenproblem was solved. We present the PCA results with the MD trajectory fitted to the first frame using $\mathrm{C}_{\alpha}$ atoms of the I and II domains. These domains are homologous in both systems. The first five eigenvectors span over $90 \%$ of the total motion of EF-G and $75 \%$ of the total motion of EF-Tu:tRNA. The amplitudes of
Fig. 2 Radius of gyration (top) and RMSD (bottom) calculated for the $\mathrm{C}_{\alpha}$ atoms of EF-G (black line) and for the $\mathrm{C}_{\alpha}$ and $\mathrm{P}$ atoms of the EF-Tu:tRNA complex (grey line) plotted as a function of the simulation time

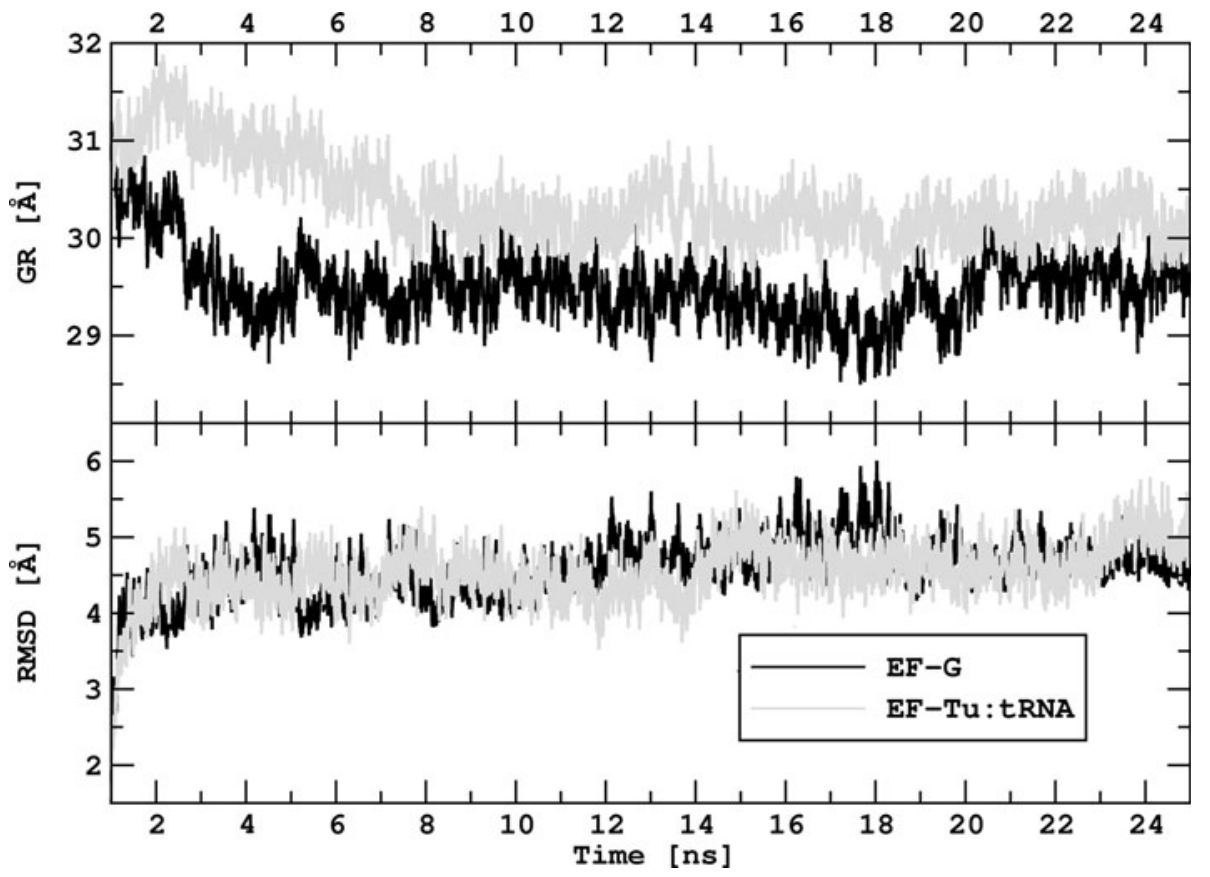


Fig. 3 RMSD matrix of EF-G (left) calculated for $\mathrm{C}_{\alpha}$ and $\mathrm{EF}-$ Tu:tRNA complex (right) calculated for $\mathrm{C}_{\alpha}$ and $\mathrm{P}$ atoms. The legend refers to the upper half of the matrices. Clusters resulting from the RMSD matrix are shown in blue below the diagonal
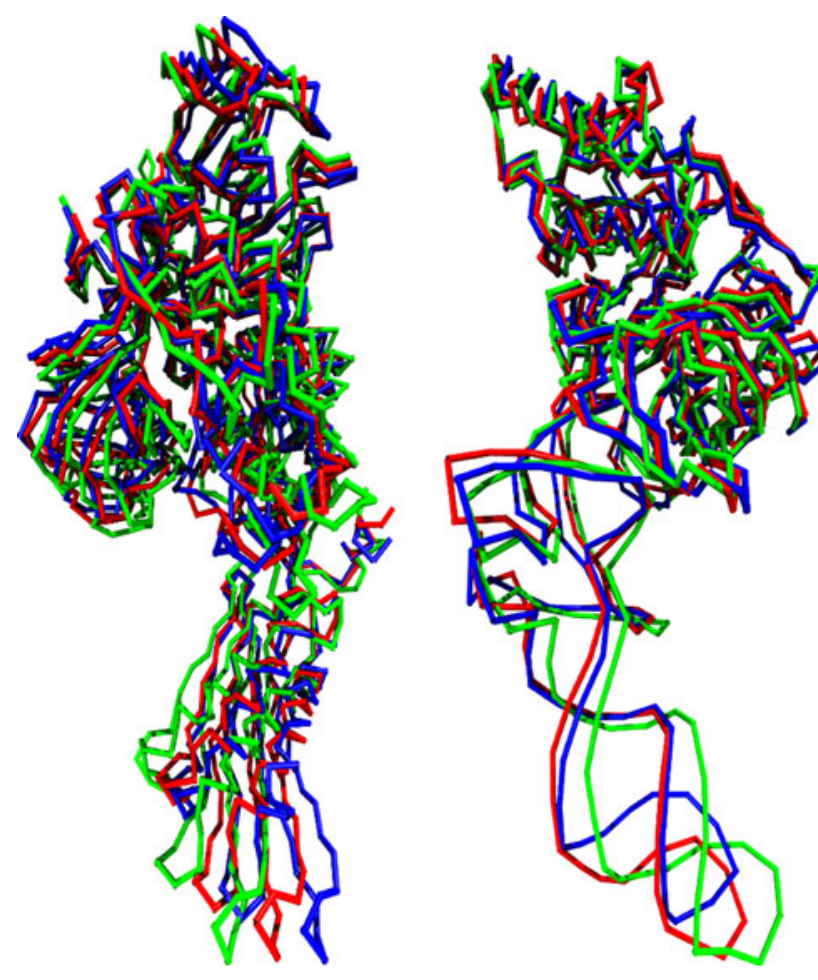

Fig. 4 Average structures of the three most numerous populations of EF-G (left) and EF-Tu:tRNA (right) derived on the basis of clustering of their RMSD matrices presented in Fig. 3

movements are comparable for both systems except for the motion along the first eigenvector. The first three vectors are presented in Fig. 6. The largest contributions are provided by the collective motions of EF-G domains III, IV, and V and tRNA of the EF-Tu:tRNA complex. For domain positions see Fig. 1.

For EF-G, PCA reveals that domains III, IV, and V undergo bending motion, occurring in different planes relative to the remaining part of the molecule; the principal vectors I and III shown in Fig. 6 (top) correspond to this motion. The observed modes corroborate with the cryo-EM maps of EF-G bound to the ribosome (Valle et al. 2003) where EF-G is present in a significantly bent configuration.

For the EF-Tu:tRNA complex the three principal vectors correspond to the stretching and bending motion of tRNA as shown in Fig. 6 (bottom). These three modes could lead to a kink of tRNA required for its proper incorporation in the A-site (Li and Frank 2007). Additionally, PCA shows that the tRNA anticodon arm can undergo extensive movement towards the D stem and, indeed, such a bent configuration was observed by cryo-EM of the ternary complex bound to the 70S ribosome (Valle et al. 2002) and in the recently determined crystal structure of EF-Tu and tRNA bound to the ribosome in the post-translocational state (Schmeing et al. 2009).

Principal directions of motion suggest that EF-G and EF-Tu:tRNA not only have homologous shapes but also show dominant movement of their corresponding extended parts, especially the EF-G domain IV and the anticodon arm of tRNA. Extensive movement of these fragments is required for the factors to properly bind to the ribosome (Wriggers et al. 2000; Tama et al. 2004; Liljas 2004 and references therein).

\section{Internal flexibility of EF-Tu and EF-Tu:GDP}

EF-Tu and EF-Tu:GDP are globular. Their radii of gyration in the MD production phase (data not shown) stabilize after 4 ns with averages of $21.8 \pm 0.3 \AA$ and $22.6 \pm 0.4 \AA$, respectively. The initial radius of gyration calculated for the crystal structure is $22 \AA$. The average RMSD of the $\mathrm{C}_{\alpha}$ atoms for EF-Tu and EF-Tu:GDP in the production phase relative to the $1 \mathrm{~ns}$ trajectory frame are $2.2 \pm 0.3 \AA$ and $3.5 \pm 0.7 \AA$, respectively. Comparison with the crystal structure gives RMSD of $3.0 \pm 0.3 \AA$ for EF-Tu and 
Fig. 5 Distances between the centres of masses (COM) of domains II and IV of EF-G (Fig. 1 left), domain II and tRNA of the EF-Tu:tRNA complex (Fig. 1, right), and domains I, II, and III of EF-Tu plotted as a function of the simulation time
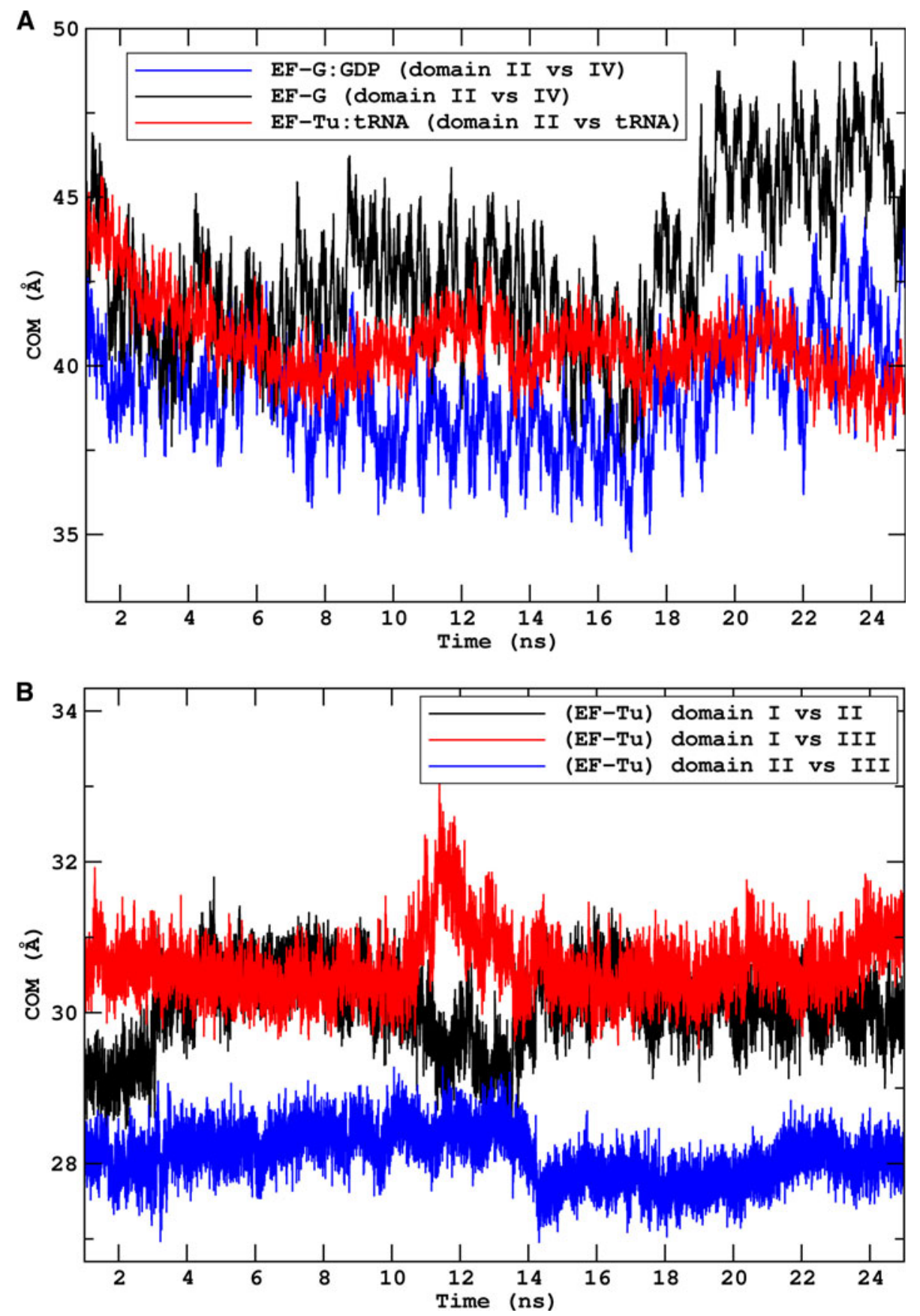

$5.4 \pm 0.6 \AA$ for EF-Tu:GDP. Larger conformational changes are thus observed for EF-Tu:GDP.

As already mentioned, for EF-Tu and EF-Tu:GDP, even though these structures are quite globular and compact, important structural differences in comparison with their crystal structures were seen. The changes occurred around the two switch regions-switch I (the socalled effector region, residues 39-65) and switch II (residues 83-100) (Nissen et al. 1995). For switch I, the $\mathrm{C}_{\alpha}$ RMSD calculated for EF-Tu:GDP changes from the initial structure by an average of $13.0 \pm 1.8 \AA$ because switch I changes its local structure (Abel et al. 1996; Polekhina et al. 1996). This switch becomes more extended and exposed to solvent compared with its conformation found in the crystal (Fig. 7). The $\alpha$-helix composed of residues 54-58 unwinds and forms a structure resembling a $\beta$-ribbon. For switch II, although no structural transitions are observed, the corresponding RMSD is $3.4 \pm 0.6 \AA$. Switch II becomes more solvent exposed because of the relative movement of protein domains (see below). These observations are in accord with earlier experiments (Abel et al. 1996; Polekhina 


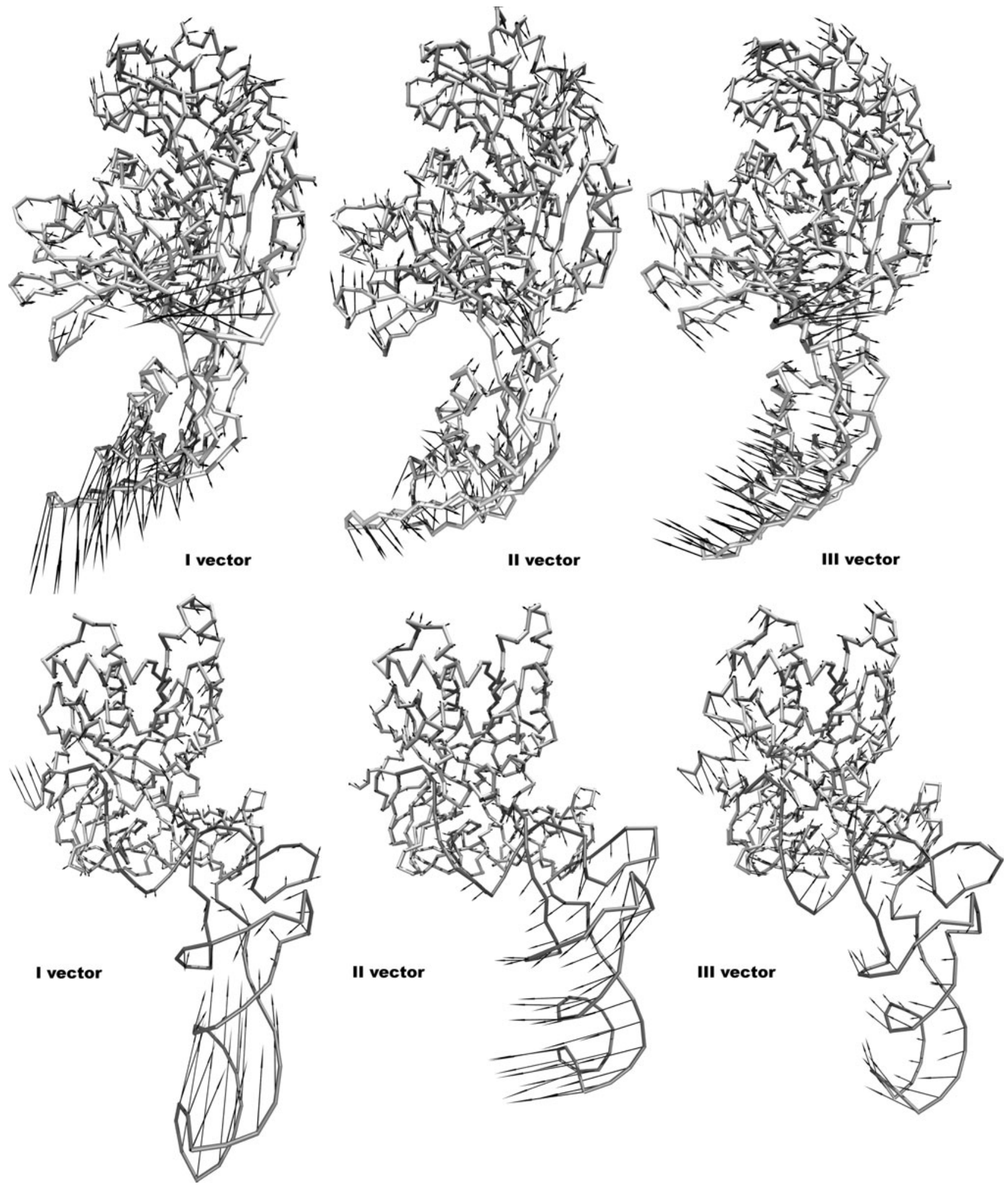

Fig. 6 Graphical representation of extreme projections along first three eigenvectors illustrating collective motion of EF-G (top) and EFTu:tRNA (bottom). Arrows show the directions of the first three eigenvectors derived from PCA

et al. 1996) which suggest that the changes in the switch I and II regions occurring in the GDP form are involved in triggering the release of EF-Tu from the ribosome.
In EF-Tu the changes of the COM distances in the MD production phase between the neighbouring domains do not exceed $4 \AA$. However, after about 12 ns of the simulation 
Fig. 7 Conformational changes observed in the switch I (in green) and II (in yellow) regions of EF-Tu. Left: Secondary structures of the switch I region of the EF-Tu crystal structure

(a) and the changes observed in MD simulations plotted as a function of the simulation time in EF-Tu (b) and EF-Tu:GDP (c). Secondary structure codes: cyan, turn; blue, 3-10 helix; yellow, extended conformation; pink, $\alpha$ helix; green, isolated bridge. Right: Representations of the X-ray structure of EF-Tu in the complex with a GTP analogue (shown in magenta as van der Waals spheres) (a), MD snapshots of EF-Tu (b) and EFTu:GDP (c); for clarity the ligand is not shown). Atom colouring: domain $\mathrm{I}(\mathrm{G})$, residues 1-211 (red); domain II, residues 220-311 (dark blue); domain III, residues 312-405 (light blue)

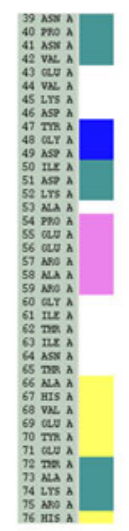

A
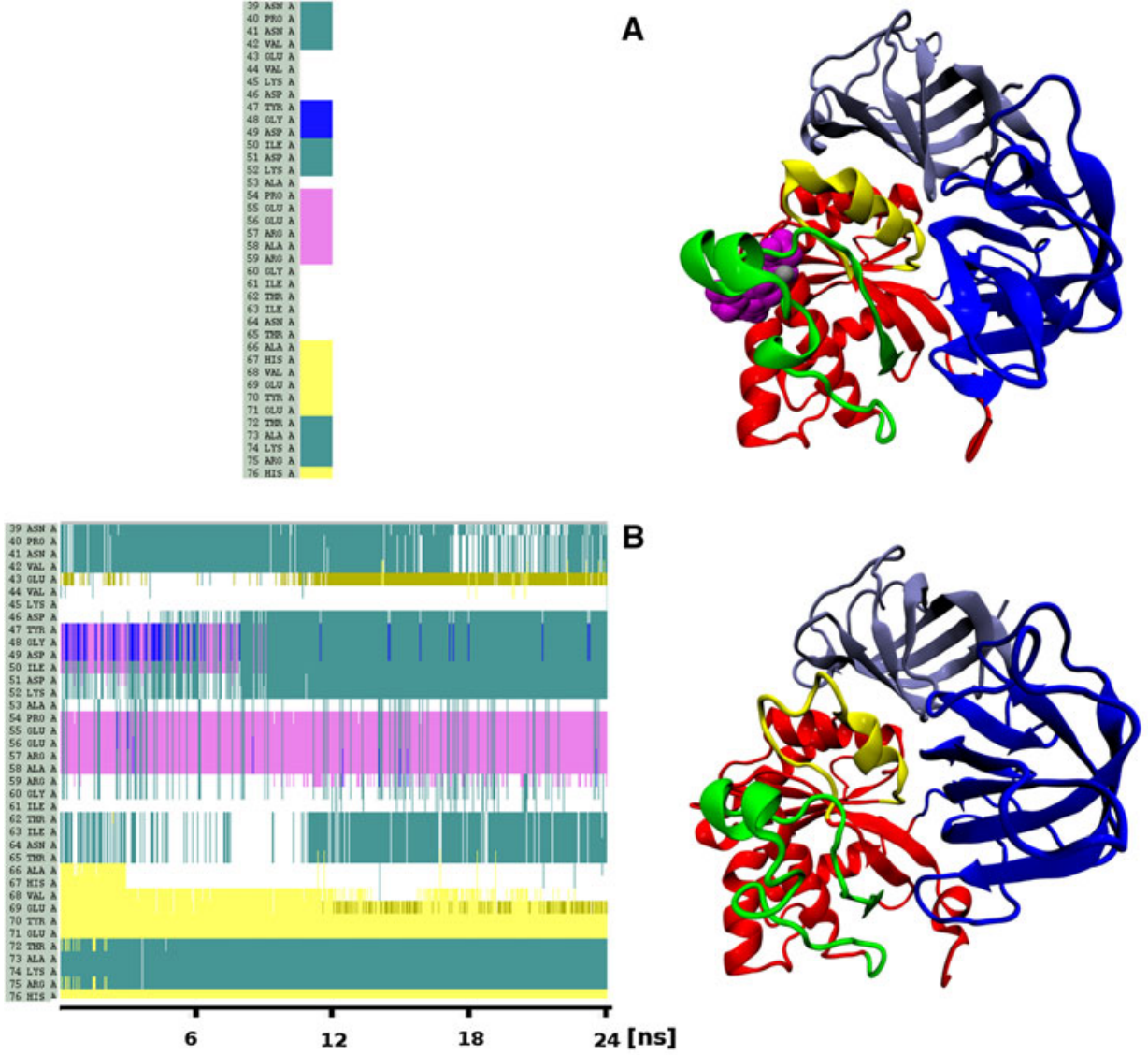

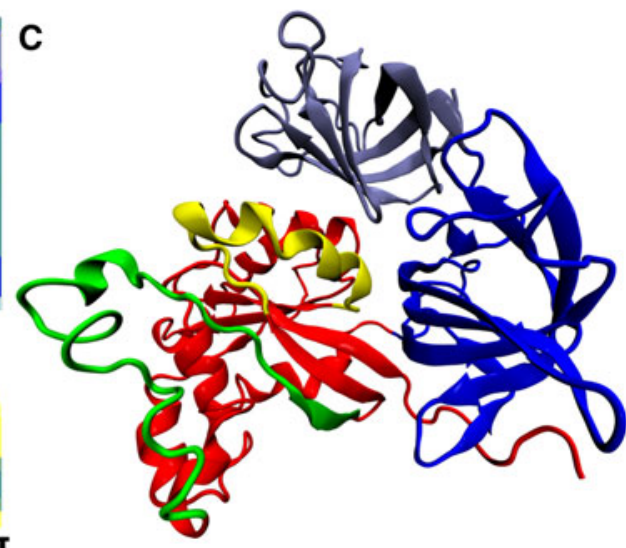

we observed reorganization of EF-Tu domains; the I domain moved toward the II domain and away from the III domain (Fig. 5b). Moreover, in comparison with the crystal structure, the relative positions of the domains of EF-Tu and EF-Tu:GDP change. However, as was observed before in the crystal structures (Polekhina et al. 1996; Abel et al. 1996; Nissen et al. 2000) the deletion of the GTP analogue or its substitution with GDP results in a movement of domains II and III relative to domain I. The position of domains II and III relative to I is important because these domains modulate the interactions of EF-Tu with various ligands (Cetin et al. 1998).

Residual fluctuations

Figure 8 shows the RMSF of $\mathrm{C}_{\alpha}$ and $\mathrm{P}$ atoms computed for the studied systems. The difference in the RMSF of the free EF-G and in the complex with GDP and $\mathrm{Mg}^{2+}$ (presented in Fig. 8a) is not substantial. This result corroborates experimental data (Liljas 2004; Hansson et al. 2005) which 
show that upon GDP or GTP analogue binding, EF-G does not significantly alter its configuration.

The RMSF differences can be seen in the EF-G switch I region (residues 38-68), which is involved in its binding to the ribosome. In EF-G:GDP, the fluctuations of switch I, which corresponds to residues located in the proximity of GDP binding site, reach $5 \AA$. In other known GTPases, the switch I loop changes its configuration depending on whether GTP or GDP is bound or the site is empty (Vetter and Wittinghofer 2001; Liljas 2004; Ticu et al. 2009). Switch I was found to be more mobile in the GDP forms of GTPases in comparison with their GTP forms (Vetter and Wittinghofer 2001). The amino acid sequence of the switch I region in EF-Tu and EF-G is highly similar. This fragment was not resolved in the crystal structures of EF-G and its initial configuration was modelled. Nevertheless, MD
Fig. 8 The root mean square fluctuation of $\mathrm{C}_{\alpha}$ and $\mathrm{P}$ atoms of a EF-G:GDP, EF-G, and b EFTu:tRNA, EF-Tu:GDP, EF-Tu
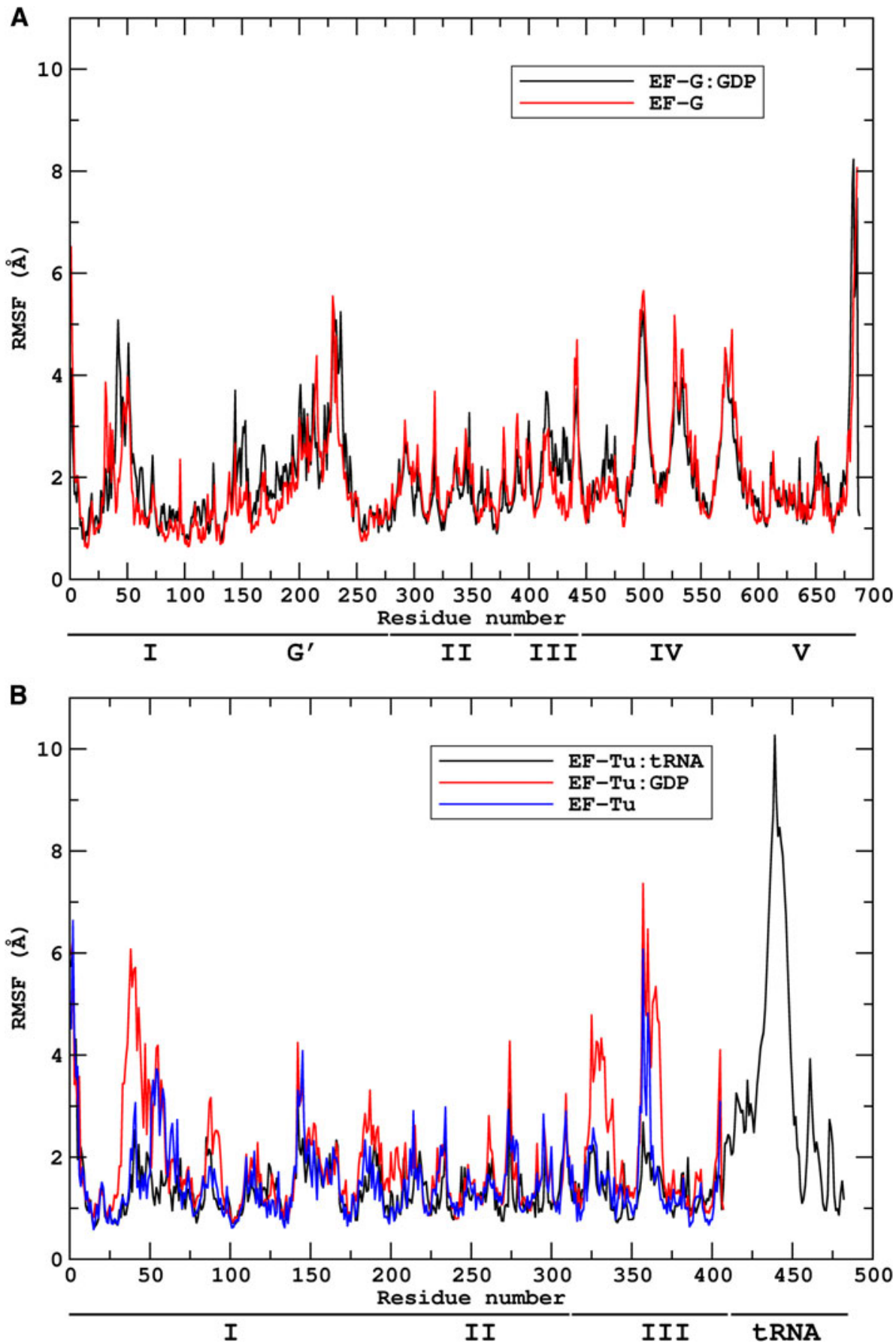
shows its flexibility which suggests the ability to undergo conformational changes similar to those occurring in switch I in other GTPases (Vetter and Wittinghofer 2001).

The EF-G and EF-G:GDP residual fluctuations are higher for domain IV, which is an extension corresponding to tRNA. Movement of domain IV is required for EF-G binding to the ribosome and during translocation of tRNAs (Gao et al. 2009). Another region with fluctuations exceeding $2 \AA$ is the $G^{\prime}$ insert (subdomain), especially the part around residue 220 that corresponds to the helix A of $\mathrm{G}^{\prime}$. This helix is exposed to solvent and was found to contact the GTPase-associated centre when EF-G binds to the ribosome (see review by Sergiev et al. 2005 and references therein). Therefore, its higher mobility observed in our MD simulations seems reasonable.

The differences in fluctuations of EF-Tu residues in the free protein, in the complex with GDP and $\mathrm{Mg}^{2+}$, and in the complex with aa-tRNA were larger than for EF-G, showing a noticeable effect of ligand binding to EF-Tu (Fig. 8b). The binding of aa-tRNA to EF-Tu reduces the RMSF mainly around residues 50-64 (the switch I region), 144-145, 276-278, and the residues of domain III. Domain III contacts the T-arm of aa-tRNA and, therefore, its movement is correlated with the presence of aa-tRNA. The fluctuations also decreased in the N-terminal region of EF$\mathrm{Tu}$ which is stabilized by interaction with aa-tRNA. The RMSF of tRNA residues are, overall, larger than that of the protein. The highest peak for tRNA can be assigned to the anticodon arm which corresponds to the tip of domain IV in EF-G. The second highest RMSF peak in tRNA corresponds to the T-arm. We also observed that pseudouridine 460 (no. 55 according to the free tRNA numbering) flips in and out of the T-arm.

For EF-Tu:tRNA we observe that the CCA-end phenylalanine ring changes its position from that observed in the crystal structure. It moves from a position between the CCA-terminus and one of the strands of the $\beta$-sheet of domain II to the inside of the protein (Fig. 9). The distances between the centres of masses of the six-membered ring of phenylalanine and the five-membered ring of His 67 in the crystal structure and in the last MD trajectory frame were 5.1 and $11.6 \AA$, respectively. The movement of the amino acid and the CCA-terminal tRNA single strand has been noticed before (Frank et al. 2005). The CCA-attached amino acid brought by tRNA must be flexible to be appropriately positioned in the peptidyl transferase centre for peptide bond synthesis (Frank et al. 2005). Also, we found that the tip of the EF-Tu switch I moves toward the tRNA acceptor stem.

The tRNA molecule needs to undergo many deformations during its entire journey through the ribosome; starting with tRNA binding to EF-Tu to form the ternary

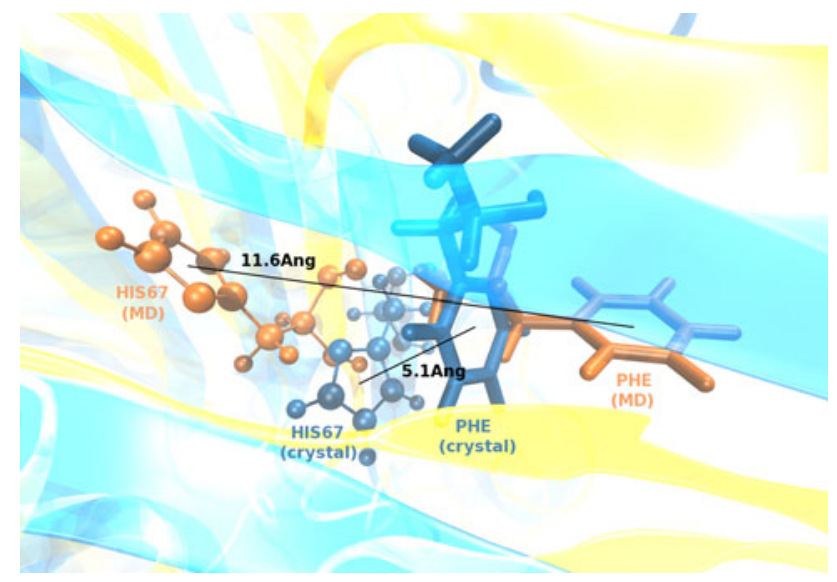

Fig. 9 The distances between the centres of masses of the CCA-end phenylalanine and histidine 67 rings observed in the crystal structure (blue) and in MD simulation (orange) of EF-Tu:tRNA

complex through correct placing of tRNA in the A-site, acquiring the $\mathrm{A} / \mathrm{T}$ transition state, translocating between tRNA binding sites, and dissociating from the E-site (see, for example, review by Agirrezabala and Frank 2009). Our MD analysis suggests that the internal mobility of tRNA is an inherent property of the EF-Tu:tRNA complex resulting from its shape and residue composition. Therefore, the ribosome only helps guide the ligands to acquire a specific configuration on the ribosome depending on the translation stage and does not force improbable or less accessible configurations.

In contrast with the EF-G case, the effect of the bound GDP and $\mathrm{Mg}^{2+}$ on the conformation of EF-Tu is substantial, which is in good agreement with experimental observations (Eargle et al. 2008). The presence of GDP and $\mathrm{Mg}^{2+}$ destabilizes EF-Tu mainly around residues 33-50, 86-93, 187-204, 261, 274, 322-338, and 356-368. Interestingly, we observe a long-distance response, as a large number of these residues is at least $17 \AA$ away from the ligand.

Electrostatic potential surfaces

The ribosome is a highly negatively charged complex and the electrostatic potential on its surface is mainly negative even though some neutral and positive patches are observed coming from the positively charged ribosomal proteins and counter-ions (Baker et al. 2001; Trylska et al. 2004; Trylska 2009). Both EF-G and EF-Tu:tRNA are negatively charged but some complementarity of the electrostatic potential of EF-G to its binding site on the ribosome was observed (Trylska et al. 2004).

Here, to compare the electrostatic surfaces of the two elongation factors, we calculated the electrostatic potential of EF-G and EF-Tu:tRNA and projected it on to their 
surfaces (Fig. 10). Figure 11 magnifies the electrostatic potential of the GTP/GDP binding region in both factors. The qualitative comparison suggests there is no global electrostatic similarity between the systems. The total charge of EF-G is -21e and that of EF-Tu:tRNA is -87e. Hence, the factors differ in the net charge by $-66 \mathrm{e}$. Also, EF-G has one more extension $\left(\mathrm{G}^{\prime}\right)$, which is not present in EF-Tu:tRNA. However, we note that under physiological conditions tRNA is partially neutralized by divalent and monovalent structural counter-ions that we are not able to explicitly include within the framework of the PoissonBoltzmann model (Grochowski and Trylska 2008).

Figure 11 shows that in both factors the GTP/GDP binding region in domain I carries a positive potential patch, which is expected because this region binds GTP or GDP that both bear a negative net charge. Domains I of EF-Tu and EF-G (Liljas 2004; March and Inouyei 1985) are homologous. However, the net charge of residues located within $5 \AA$ of the GDP heavy atoms is different. For EF-G, the GDP/GTP binding cleft is composed of two Asp, four Lys, and one Arg residue. Assuming their protonation states as in solution at $\mathrm{pH} 7$ the total charge is +3 e. In EF-Tu:tRNA, the ionisable residues in the proximity of GDP/GTP are two Asp and two Lys which, overall, give a neutral charge. The residues that are in structurally corresponding positions in relation to GDP/ GTP in both factors are: Asp22, Lys25, Lys138, Asp140 in EF-G and, respectively, Asp21, Lys24, Lys137, Asp139 in EF-Tu.

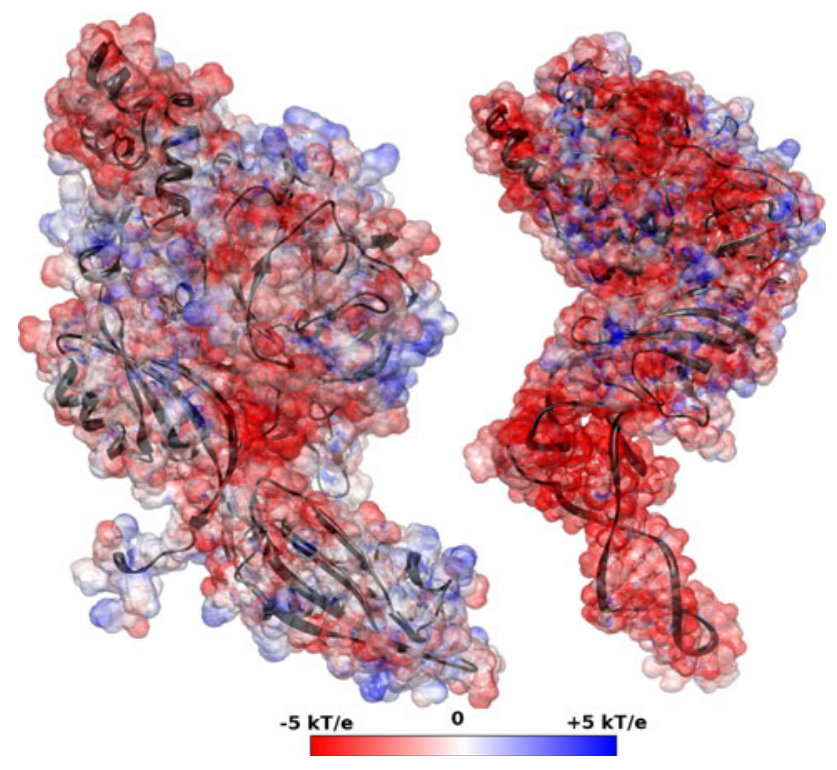

Fig. 10 The electrostatic potential of EF-G (left) and the EFTu:tRNA complex (right) projected on to their van der Waals surfaces (partially transparent). Interior black detail shows the secondary structure of EF-G and EF-Tu:tRNA

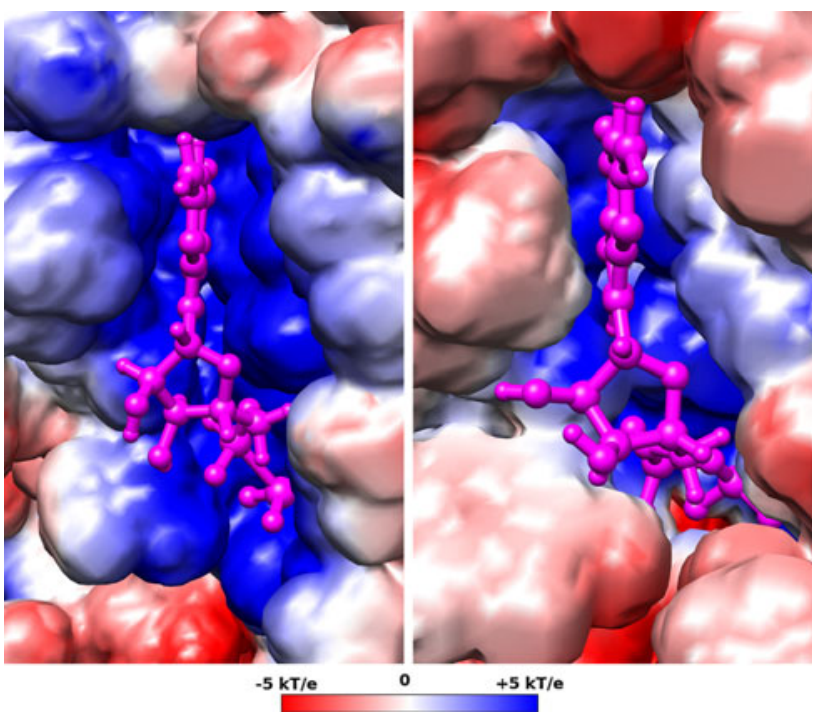

Fig. 11 The electrostatic potential in the GTP/GDP binding site (GDP is shown in purple) projected on to van der Waals surfaces of EF-G (left) and EF-Tu:tRNA (right)

A part of domain I of EF-G and EF-Tu, which binds to the $\mathrm{L} 11$ protein on the ribosome, has a negative potential (Fig. 10). EF-G domain IV has a neutral or positive potential as opposed to tRNA in the EF-Tu:tRNA complex. The positive potential regions in the tip of the domain IV were also observed in our earlier study (Trylska et al. 2004). If both factors bind to the same site on the ribosome, the involvement of divalent ions around tRNA and surrounding solvent is expected to neutralize the charge of tRNA. Because the ribosomal GTPase associated centre acquires either an open or a closed conformation depending on whether EF-G or ternary complex are bound (Sergiev et al. 2005), the factors "feel" a slightly different electrostatic response from the ribosome. Therefore, we conclude that the EF-G and EF-Tu:tRNA binding site on the ribosome is more shape than electrostatics-specific and that the ribosome can acquire a conformation that is also electrostatically complementary toward a specific elongation factor.

EF-Tu is a negatively charged protein $(-14 \mathrm{e})$ and binds a negatively charged aa-tRNA. We calculated the electrostatic potential around the EF-Tu and analysed the regions in the vicinity of domains II and III that bind aa-tRNA. The projection of the electrostatic potential on the EF-Tu surface is presented in Fig. 12. Positive potential patches exist in the areas of binding of the T-arm (see also Fig. 1) and the $5^{\prime}$ and $3^{\prime}$ termini of tRNA. However, there is no global and continuous positive potential area from the side of the tRNA binding. Nevertheless, both non-catalytic domains of EF-Tu are important for tRNA binding because it was found that deletion of either domain II or III weakens the interactions of EF-Tu with tRNA (Cetin et al. 1998). 


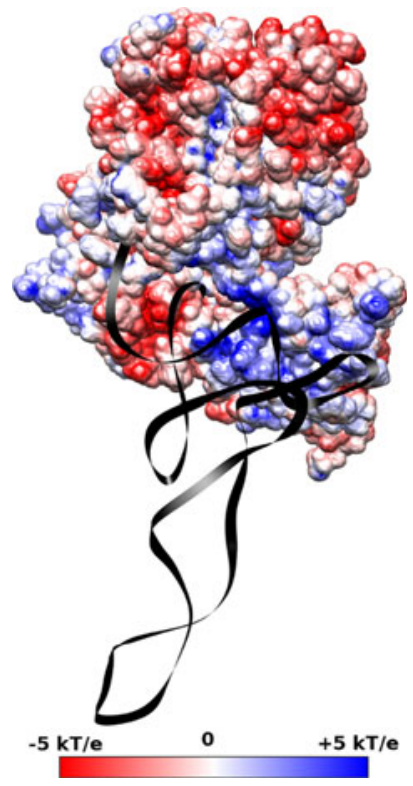

Fig. 12 The electrostatic potential calculated for the isolated EF-Tu projected on to its van der Waals surface. The tRNA molecule (black ribbon) is also shown to present its binding mode

\section{Conclusions}

We have presented the results of all-atom MD simulations performed for the bacterial ribosomal elongation factors EF$\mathrm{G}$ and EF-Tu. The main objective of the study was to compare their internal flexibility. MD simulations were performed with a generalized Born (Onufriev et al. 2004) approach for modelling of the solvation effects. The same and similar approaches have been widely used to investigate the flexibility of biomolecules (Mongan et al. 2004; Srinivasan et al. 1998; Formaneck and Cui 2006), and to study protein folding (Bursulaya and Brooks 2000; Lei et al. 2007). For example, Formaneck and Cui (2006) showed good agreement of the results obtained using the explicit and implicit solvent models for small proteins. Bursulaya and Brooks (2000) applied generalized Born and TIP3P explicit water models to study the folding of a three-stranded $\beta$-sheet protein Betanova. They have shown that an implicit solvent model was adequate for such studies. Williams and Hall (1999) and Sorin et al. (2002) used a generalized Born implicit solvent model to investigate nucleic acids and the results were in agreement with an explicit solvent model and experiments. We are aware, however, that the implicit solvation model used in this work is only an approximation to the exact solution of the linearised Poisson-Boltzmann equation (Honig and Nicholls 1995). Its performance for highly charged proteins and nucleic acids might be imperfect because the solutions of the linear form of the PoissonBoltzmann equation can deviate from those obtained with its full, nonlinear form. While there are generalized Born models that enable more accurate modelling of electrostatic effects for nucleic acids and highly charged proteins (Tjong and Zhou 2007), unfortunately their implementation in molecular dynamics is still lacking.

EF-G and EF-Tu:tRNA are examples of molecularmimicry and our study demonstrated that their overall dynamics, also, are slightly similar. The largest contribution to the mobility of the factors comes from their extensions or tails (i.e. domain IV of EF-G and aa-tRNA of the EF-Tu:tRNA complex). These fragments have similar motion that leads to their proper positioning on the ribosome with tips of domain IV and tRNA placed in the Asite. MD simulations also showed that EF-Tu in the complex with GDP acquires a different configuration than in the crystal structure with a GTP analogue. Domains II and III move relative to domain I which corroborates experimental data (Nissen et al. 2000). We observed a change of local structure in the switch I region of EF-Tu. We also compared the electrostatic properties of EF-G and EFTu:tRNA and found no global electrostatic similarity on their surface. However, some corresponding parts, especially the GTP/GDP binding region and domain I, have patches of similar electrostatic potential. Therefore, we conclude that the binding of EF-G and the ternary complex to the ribosome is more shape than electrostatic-specific.

Acknowledgments We acknowledge support from the University of Warsaw (ICM BST 1255/2008, 1450/2009, 1550/2010 and G31-4), Polish Ministry of Science and Higher Education (N N301 245236), the Fogarty International Center (NIH Research Grant R03 TW07318), and the Foundation for Polish Science (Focus program and TEAM/2009-3/8 - co-financed by European Regional Development Fund operated within Innovative Economy Operational Programme). KK is supported by the EU through the European Social Fund, contract 146 number UDA-POKL.04.01.01-00-072/09-00.

Open Access This article is distributed under the terms of the Creative Commons Attribution Noncommercial License which permits any noncommercial use, distribution, and reproduction in any medium, provided the original author(s) and source are credited.

\section{References}

Abel K, Yoder MD, Hilgenfeld R, Jurnak F (1996) A complex profile of protein elongation: translating chemical energy into molecular movement. Structure 4:229-238

Agirrezabala X, Frank J (2009) Elongation in translation as a dynamic interaction among the ribosome, tRNA, and elongation factors EF-G and EF-Tu. Q Rev Biophys 42:159-200

Agrawal RK, Penczek P, Grassucci RA, Frank J (1998) Visualization of elongation factor $\mathrm{G}$ on the Escherichia coli $70 \mathrm{~S}$ ribosome: the mechanism of translocation. Proc Natl Acad Sci USA 95:6134-6138

Agrawal RK, Heagle AB, Penczek P, Grassucci RA, Frank J (1999) EF-G-dependent GTP hydrolysis induces translocation accompanied by large conformational changes in the $70 \mathrm{~S}$ ribosome. Nat Struct Biol 6:643-647 
Amadei A, Linssen ABM, Berendsen HJC (1993) Essential dynamics of proteins. Proteins Struct Funct Genet 17:412-425

Andrea TA, Swope WC, Andersen HC (1983) The role of long ranged forces in determining the structure and properties of liquid water. J Chem Phys 79:4576-4584

Baker NA, Sept D, Joseph S, Holst MJ, McCammon JA (2001) Electrostatics of nanosystems: application to microtubules and the ribosome. Proc Natl Acad Sci USA 98(18):10037-10041

Berendsen HJC, van der Spoel D, van Drunen R (1995) GROMACS: a message passing parallel molecular dynamics implementation. Comput Phys Comm 91:43-56

Bursulaya BD, Brooks CL III (2000) Comparative study of the folding free energy landscape of a three-stranded $\beta$-sheet protein with explicit and implicit solvent models. J Phys Chem B 104:12378-12383

Case DA, Cheatham TE III, Darden T, Gohlke H, Luo R, Merz KM, Onufriev A, Simmerling C, Wang B, Woods RJ (2005) The Amber biomolecular simulation programs. J Comput Chem 26:1668-1688

Case DA, Darden TA, Cheatham III TE, Simmerling CL, Wang J, Duke RE, Luo R, Merz KM, Pearlman DA, Crowley M, Walker RC, Zhang W, Wang B, Hayik S, Roitberg A, Seabra G, Wong KF, Paesani F, Wu X, Brozell S, Tsui V, Gohlke H, Yang L, Tan C, Mongan J, Hornak V, Cui G, Beroza P, Mathews DH, Schafmeister C, Ross WS, Kollman PA (2006) Amber 9. University of California, San Francisco

Cetin R, Anborgh PH, Cool RH, Parmeggiani A (1998) Functional role of the noncatalytic domains of elongation factor $\mathrm{Tu}$ in the interactions with ligands. Biochemistry 37:486-495

Czworkowski J, Wang J, Steitz TA, Moore PB (1994) The crystal structure of elongation factor G complexed with GDP, at $2.7 \AA$ resolution. EMBO J 13:3661-3668

Duan Y, Wu C, Chowdhury S, Lee MC, Xiong G, Zhang W, Yang R, Cieplak P, Luo R, Lee T, Caldwell J, Wang J, Kollman P (2003) A point-charge force field for molecular mechanics simulations of proteins based on condensed-phase quantum mechanical calculations. J Comput Chem 24:1999-2012

Eargle J, Black AA, Sethi A, Trabuco LG, Luthey-Schulten Z (2008) Dynamics of recognition between tRNA and elongation factor Tu. J Mol Biol 377:1382-1405

Formaneck MS, Cui Q (2006) The use of a generalized born model for the analysis of protein conformational transitions: a comparative study with explicit solvent simulations for chemotaxis Y protein (CheY). J Comput Chem 27:1923-1943

Frank J, Agrawal RK (2000) A ratchet-like inter-subunit reorganization of the ribosome during translocation. Nature 406:318-322

Frank J, Sengupta J, Gao H, Li W, Valle M, Zavialovd A, Ehrenbergd $\mathrm{M}$ (2005) The role of tRNA as a molecular spring in decoding, accommodation, and peptidyl transfer. FEBS Lett 579:959-962

Gao YG, Selmer M, Dunham CM, Weixlbaumer A, Kelley AC, Ramakrishnan V (2009) The structure of the ribosome with elongation factor $G$ trapped in the posttranslocational state. Science 326:694-699

Grochowski P, Trylska J (2008) Continuum molecular electrostatics, salt effects and counterion binding. A review of the PoissonBoltzmann model and its modifications. Biopolymers 89:93-113

Gudkov AT (2001) Structure and function of the prokaryotic elongation factor G. J Mol Biol 35(4):552-558

Hansson S, Singh R, Gudkov AT, Liljas A, Logan DT (2005) Crystal structure of a mutant elongation factor G trapped with a GTP analogue. FEBS Lett 579:4492-4497

Honig B, Nicholls A (1995) Classical electrostatics in biology and chemistry. Science 268:1144-1149

Humphrey W, Dalke A, Schulten K (1996) VMD—visual molecular dynamics. J Mol Graph 14:33-38
Jarvis RA, Patrick EA (1973) Clustering using a similarity measure based on shared near neighbors. IEEE Trans Comput 22(11): $1025-1034$

Jeppesen MG, Navratil T, Spremulli LL, Nyborg J (2005) Crystal structure of the bovine mitochondrial elongation factor $\mathrm{Tu} \mathrm{Ts}$ complex. J Biol Chem 280:5071-5081

Johanson U, AEvarsson A, Liljas A, Hughes D (1996) The dynamic structure of EF-G studied by fusidic acid resistance and internal revertants. J Biol Chem 258:420-432

Kawashima T, Berthet-Colominas C, Wulff M, Cusack S, Leberman R (1996) The structure of the Escherichia coli EF-Tu:EF-Ts complex at $2.5 \AA$ resolution. Nature 379:511-518

Kjeldgaard M, Nissen P, Thirup S, Nyborg J (1993) The crystal structure of elongation factor EF-Tu from Thermus aquaticus in the GTP conformation. Structure 1(1):35-50

Laurberg M, Kristensen O, Martemyanov K, Gudkov AT, Nagaev I, Hughes D, Liljas A (2000) Structure of a mutant EF-G reveals domain III and possibly the fusidic acid binding site. J Mol Biol 303:593-603

Lee MC, Duan Y (2004) Distinguish protein decoys by using a scoring function based on a new amber force field, short molecular dynamics simulations, and generalized born solvent model. Proteins Struct Funct Bioinf 55:620-634

Lei H, Wu C, Liu H, Duan Y (2007) Folding free-energy landscape of villin headpiece subdomain from molecular dynamics simulations. Proc Natl Acad Sci USA 104:4925-4930

Li W, Frank J (2007) Transfer RNA in the hybrid P/E state: correlating molecular dynamics simulations with cryo-EM data. Proc Natl Acad Sci USA 29:16540-16545

Liljas A (2004) Structural aspects of protein synthesis. World Scientific, Singapore

March PE, Inouyei M (1985) GTP-binding membrane protein of Escherichia coli with sequence homology to initiation factor 2 and elongation factors Tu and G. Proc Natl Acad Sci USA 82:7500-7504

Martemyanov KA, Gudkov AT (1999) Domain IV of elongation factor $\mathrm{G}$ from Thermus thermophilus is strictly required for translocation. FEBS Lett 452:155-159

Martemyanov KA, Gudkov AT (2000) Domain III of elongation factor $\mathrm{G}$ from Thermus thermophilus is essential for induction of GTP hydrolysis on the ribosome. J Biol Chem 275:35820-35824

Mongan J, Case DA, McCammon JA (2004) Constant pH molecular dynamics in generalized born implicit solvent. J Comput Chem 25:2038-2048

Munro JB, Altman RB, Tung CS, Sanbonmatsu KY, Blanchard SC (2010) A fast dynamic mode of the ef-g-bound ribosome. EMBO J 29:770-781

Nissen P, Kjeldgaard M, Thirup S, Polekhina G, Reshetnikova L, Clark BFC, Nyborg J (1995) Crystal structure of the ternary complex of Phe-tRNA, EF-Tu, and a GTP analog. Science 270:1464-1472

Nissen P, Kjeldgaard M, Nyborg J (2000) Macromolecular mimicry. EMBO J 19:489-495

Nyborg J, Nissen P, Kjeldgaard M, Thirup S, Polakhina G, Clark BFC, Reshetnikova L (1996) Structure of the ternary complex of EF-Tu: macromolecular mimicry in translation. Trends Biochem Sci 21(3):81-82

Onufriev A, Bashford D, Case DA (2004) Exploring protein native states and large-scale conformational changes with a modified generalized Born model. Proteins Struct Funct Bioinf 55:383-394

Pearlman DA, Case DA, Caldwell JW, Ross WS, Cheatham TE, DeBolt S, Ferguson D, Seibel G, Kollman P (1995) AMBER, a package of computer programs for applying molecular mechanics, normal mode analysis, molecular dynamics and free energy 
calculations to simulate the structural and energetic properties of molecules. Comput Phys Commun 91:1-41

Pettersen EF, Goddard TD, Huang CC, Couch GS, Greenblatt DM, Meng EC, Ferrin TE (2004) UCSF chimera-a visualization system for exploratory research and analysis. J Comput Chem 25(13):1605-1612

Polekhina G, Thirup S, Kjeldgaard M, Nissen P, Lippmann C, Nyborg $\mathrm{J}$ (1996) Helix unwinding in the effector region of elongation factor EF-Tu-GDP. Structure 4:1141-1151

Rodnina MV, Fricke R, Kuhn L, Wintermeyer W (1995) Codondependent conformational change of elongation factor Tu preceding GTP hydrolysis on the ribosome. EMBO J 14:2613-2619

Ryckaert JP, Ciccotti G, Berendsen HJC (1977) Numerical integration of the cartesian equations of motion of a system with constraints: Molecular dynamics of n-alkanes. J Comput Phys 23(3):327-341

Sanbonmatsu KY, Joseph S, Tung CS (2005) Simulating movement of tRNA into the ribosome during decoding. Proc Natl Acad Sci USA 44:15854-15859

Schmeing TM, Voorhees RM, Kelley AC, Gao YG, Murphy FV IV, Weir JR, Ramakrishnan V (2009) The crystal structure of the ribosome bound to EF-Tu and aminoacyl-tRNA. Science 326:688-694

Schuette JC, Murphy FV IV, Kelley AC, Weir JR, Giesebrecht J, Connell SR, Loerke J, Mielke T, Zhang W, Penczek PA, Ramakrishnan V, Spahn CMT (2009) GTPase activation of elongation factor EF-Tu by the ribosome during decoding. EMBO J 28:755-765

Sergiev PV, Bogdanov AA, Dontsova OA (2005) How can elongation factors EF-G and EF-Tu discriminate the functional state of the ribosome using the same binding site? FEBS Lett 579: 5439-5442

Shen M, Freed KF (2002) Long time dynamics of met-enkephalin: comparison of explicit and implicit solvent models. Biophys $\mathrm{J}$ 82:1791-1808

Sorin EJ, Engelhardt MA, Herschlag D, Pande VS (2002) RNA simulations: probing hairpin unfolding and the dynamics of a GNRA tetraloop. J Mol Biol 317:493-506

Spasic A, Sitha M, Chu S, Mohanty U (2008) Polyelectrolyte behavior and kinetics of aminoacyl-tRNA on the ribosome. J Phys Chem B 112:4161-4163

Srinivasan J, Cheatham TE, Cieplak P, Kollman PA, Case DA (1998) Continuum solvent studies of the stability of DNA, RNA, and
phosphoramidate-DNA helices. J Am Chem Soc 120:9401-9409

Stark H, Rodnina MV, Wieden HJ, van Heel M, Wintermeyer W (2000) Large-scale movement of elongation factor $G$ and extensive conformational change of the ribosome during translocation. Cell 100:301-309

Tama F, Miyashita O, Brooks CL III (2004) Normal mode based flexible fitting of high-resolution structure into low-resolution experimental data from cryo-EM. J Struct Biol 147:315-326

Ticu C, Nechifor R, Nguyen B, Desrosiers M, Wilson KS (2009) Conformational changes in switch $\mathrm{i}$ of ef-g drive its directional cycling on and off the ribosome. EMBO J 28:2053-2065

Tjong H, Zhou HX (2007) GBr6NL: a generalized Born method for accurately reproducing solvation energy of the nonlinear Poisson-Boltzmann equation. J Chem Phys 126:195102

Trylska J (2009) Simulating activity of the bacterial ribosome. Q Rev Biophys 42:301-316

Trylska J, Konecny R, Tama F, Brooks CL III, McCammon JA (2004) Ribosome motions modulate electrostatic properties. Biopolymers 74:423-431

Tsui V, Case DA (2000) Molecular dynamics simulations of nucleic acids with a generalized born solvation model. J Am Chem Soc 122:2489-2498

Valle M, Sengupta J, Swami NK, Grassucci RA, Burkhardt N, Nierhaus KH, Agrawal RK, Frank J (2002) Cryo-EM reveals an active role for aminoacyl-tRNA in the accommodation process. EMBO J 21:3557-3567

Valle M, Zavialov A, Sengupta J, Rawat U, Ehrenberg M, Frank J (2003) Locking and unlocking of ribosomal motions. Cell 114:123-134

Vetter IR, Wittinghofer A (2001) The guanine nucleotide-binding switch in three dimensions. Science 294:1299-1304

Vogeley L, Palm GJ, Mesters JR, Hilgenfeld R (2001) Conformational change of elongation factor $\mathrm{Tu}$ (EF-Tu) induced by antibiotic binding. J Biol Chem 276:17149-17155

Williams DJ, Hall KB (1999) Unrestrained stochastic dynamics simulations of the UUCG tetraloop using an implicit solvation model. Biophys J 76:3192-3205

Wriggers W, Agrawal RK, Drew DL, McCammon JA, Frank J (2000) Domain motions of EF-G bound to the 70S ribosome: Insights from a hand-shaking between multi-resolution structures. Biophys J 79:1670-1678 